\title{
Radiation, a two-edged sword: From untoward effects to fractionated radiotherapy
}

\author{
M.T. Chew ${ }^{\mathrm{a}, *}$, B. Jones ${ }^{\mathrm{b}}$, M. Hill ${ }^{\mathrm{b}}$, D.A. Bradley ${ }^{\mathrm{a}, \mathrm{c}}$ \\ ${ }^{a}$ Centre for Biomedical Physics, School of Healthcare and Medical Sciences, Sunway University, No. 5, Jalan Universiti, Bandar Sunway, 47500, Selangor Darul Ehsan, \\ Malaysia \\ ${ }^{\mathrm{b}}$ CRUK/MRC Oxford Institute for Radiation Oncology, Department of Oncology, University of Oxford, ORCRB Roosevelt Drive, Oxford, OX3 7DQ, United Kingdom \\ ${ }^{\mathrm{c}}$ Department of Physics, Faculty of Engineering and Physical Sciences, University of Surrey, Guildford, GU2 7XH, United Kingdom
}

\section{A R T I C L E I N F O}

\section{Keywords:}

Two-edged sword

Radiobiological effect

Chromosomal aberrations

6 Rs of Radiobiology

\begin{abstract}
A B S T R A C T
Radiations in medicine cover a wide range of applications, predominantly in diagnostic imaging and radiotherapy, encompassing photons ( $\mathrm{x}$ - and $\gamma$-rays) and particle radiation, as well as with the use of liquid sources in nuclear medicine focusing on physiological functional imaging, tumour detection or targeted radiotherapy. The biological interactions of ionizing radiation leads naturally to questions of benefits and risk following dose exposures. The inherent properties of ionizing radiation in sterilising dividing cells can offer immense benefits with respect to tumour control, but radiation can also deliver potential harm in the form of normal tissue toxicity or carcinogenesis. The advances in radiation technology, offering accurate and reliable dose delivery, in concert with greater understanding of the underpinning radiobiological effects are creating an ever-growing ability to extract maximum benefit and minimise risk. The radiobiological effects fall broadly under the headings of mutagenesis, chromosomal aberrations, radiation induced genomic instability and cell death. The enormity of evidence derived from these underlie the mechanism of the six Rs of controlled radiotherapy: repair, repopulation, reoxygenation, redistribution, radiosensitivity and most recently, remote bystander cellular effects (including low dose hyper-radiosensitivity, adaptive response, hormesis, abscopal effect and immune response). Herein, we seek to discuss how such understanding leads to optimised radiotherapy.
\end{abstract}

\section{Introduction}

In the broadest of terms, medical radiations are accessed for diagnosis and radiotherapy, with the use of photons ( $\mathrm{x}$ - and $\gamma$-rays) and particle radiation (proton and carbon) well-embedded in routine medical centre practices. Ionizing radiation interactions within the human body arise from external sources, also from radioactive isotopes introduced internally, offering great benefits in specific medical circumstances, but with the capacity to cause harm if used inappropriately or incorrectly. Understanding of the science-base is essential for optimisation purposes.

Ionizing radiation deposits energy in highly structured tracks of ionization and excitation events, with the general structure of these tracks varying for the different types of radiation, resulting in differences in biologically effectiveness for a given dose (Hill, 2019). Linear energy transfer (LET) is often used to describe the average rate of energy loss along the track of an ionizing particle. Accordingly, different forms of radiation can be classified as either sparsely ionizing low LET radiations (such as photons and electrons) or more densely ionizing high LET (such as alpha-particles and carbon ions), although there is inevitably a spectrum of LET values.

Radiotherapy relies on molecular damage produced by ionizing radiation, which can occur either through direct interaction or indirectly as a result of interaction with the surrounding water (Hall and Giaccia, 2012). Directly ionizing radiation has sufficient kinetic energy to disrupt particular atomic structures within DNA in the tissue through which they pass, producing chemical and biological changes. Ionizing radiation has the ability to remove tightly bound electrons from their atomic orbits, causing the atom to become charged, more commonly termed ionized. This ionized atom can then react with neighbouring atoms and ions forming new chemical bonds. In tissue, the energy released by an ionizing event is typically of the order of $34 \mathrm{eV}$ in creating an ion pair, sufficient to break strong chemical bonds, such as the $\mathrm{C}=\mathrm{C}$ bond with an associated energy of $4.9 \mathrm{eV}$ (Hall and Giaccia, 2012). The charged atom will further interact with several atoms or molecules. Ionization events in the surrounding of water can result in the release of

\footnotetext{
* Corresponding author.

E-mail address: mtchew@sunway.edu.my (M.T. Chew).
} 
highly reactive free radicals, most notably hydroxyl radicals which are able to diffuse and react with DNA.

Radiotherapy and radiosurgery are important in the medical management of a large range of conditions that manifest in dysfunction resulting from changes in tissue and tissue response (structure, organisation, cellular division etc.), cancer in particular. The basis of radiation therapy is to deposit sufficient energy via atomic ionizations to eliminate the tumour cells, while sparing as much as possible the normal tissues or organs that surround a tumour. Ideally, the greater the absorbed energy (or radiation dose) to the tumour tissue, the greater the probability of lethality to the tumour. Hence, the technical ability to localise and maximise energy deposition within a well-defined volume (the tumour and its normal tissue margin) is of paramount importance in the delivery of radiotherapy. The efficacy of radiation therapy is further improved by recent technological and biological advancements and developments. The advances in technology include improved physical delivery of beams and imaging, with better-quality dose distribution, for instance from use of intensity modulated and image guided radiotherapy (IMRT and IGRT), stereotactic radiosurgery, stereotactic body radiotherapy (SRBT) and many others - resulting in maximised dose to the tumour and minimised dose to surrounding normal tissues. These advances in radiotherapy planning and delivery, with accurate targeting of radiation to the tumour have facilitated greater conformity, reduced planning margins and the delivery of an increased dose to the tumour, potentially resulting in improved patient outcomes.

These advances taking place in radiation technology, not least accurate dose delivery via precise measures of control, in concert with greater understanding of the underpinning radiobiological effects are creating an ever-growing ability to extract maximum benefit while minimising the risk of harm.

The radiobiological effects of ionizing radiations fall broadly under the headings of cell death, mutagenesis, chromosomal aberrations, radiation induced genomic instability. The enormity of evidence derived from these underlie the so-called six Rs of controlled radiotherapy: repair, repopulation, reoxygenation, redistribution, radiosensitivity and most recently, remote bystander cellular effects (including low dose hypersensitivity, adaptive response, hormesis, abscopal effects and immune response). How such understanding can lead to optimised radiotherapy is described below.

\section{Radiobiology}

\subsection{Effects of radiation in cells - Damage, repair and cell death}

The impact of radiation on living human cells/tissues varies with the radiation modality and the differing types and properties of tissues. Moreover, ionizing radiation generates chemical, biological, biochemical and physiological changes, succinctly described by JB Little (1968) as shown in Fig. 1 (Little, 1968). Chemically, each deposition of $34 \mathrm{eV}$ is sufficient to produce an ion pair and this can trigger a cascade of mechanisms that can contribute to cell death with increasing dose. Biologically, ionizing radiation induces several basic types of response from the cells such as oxidative stress, activating and inactivating of different signalling pathways, and damage to deoxyribonucleic acid (DNA); the latter include base loss, base modification, dimer, singleand double-strand breaks (Hill, 2019). Double strands break (DSB) are considered the most lethal form of DNA damage. A percentage of these DSBs will have additional strand-breaks and/or based damage within a few base pairs, and are referred to as complex DSBs, which are much more difficult to repair than simple DSB. While typically these DSBs will be quickly repaired by the cell, miss-repair can result in mutations, and alternatively broken ends may undergo illegitimate re-joining with other nearby DSB resulting in chromosomal aberrations, which may or may not be lethal. If the cell survives, there is a risk of future cancer development. Besides the DSBs, radiation does directly damage the atomic structures of proteins and lipids (Pouget et al., 2018) and interferes with membrane function, although these may be temporary changes since a viable cell is capable of replacing damaged macromolecules. Such radiation effects do activate a series of downstream signalling effects that may even persist for an extended period of time following irradiation; this can result in a variety of responses including cell death. Cell death can be triggered by apoptosis, necrosis, mitotic catastrophe, autophagy and all these events could occur concurrently and are dependent on the cell type. Cells that undergo apoptosis are typically significantly more radiosensitive then cells which predominantly die as a result of mitotic catastrophe (Hall and Giaccia, 2012). Tumour cell death is beneficial but cellular and vascular tissue damage in the surrounding normal tissue or organs can be detrimental.

Mammalian cells also have the ability to repair radiation damage by means of complex enzymatic systems. Both normal and tumour cells have the ability to repair radiation induced damage. Due to gene mutation, some tumour cells appear to repair faster than normal cells, probably due to functional loss of repair systems which have longer half-times of repair, and also may repair with reduced fidelity.

The three different type of induced radiation damage are: (i) lethal damage which are irreversible and irreparable and that lead to cell death (Hall and Kraljevic, 1976; Hall and Giaccia, 2012); (ii) sublethal damage (Elkind and Sutton, 1959); and (iii) potentially lethal damage (PLD) (Phillips and Tolmach, 1966). These three types of damage are related to DNA single and DSBs which are the basic lesions responsible for further chromosomal injury and radiation-induced cell inactivation. In human cells, radiation induced DNA DSBs spontaneously trigger a DNA damage response (DDR) process. The DDR process comprises of a complex network of proteins that initiate and coordinate DNA damage signalling and repair activities. DNA repair depends on several factors, such as types of damage, cell cycle phase of cell (kinases and cell cycle check points) and repair pathways. For single-strand break (SSB), the three modes of excision repair are Base Excision Repair, Nucleotide Excision Repair and Mismatch Repair. The two major pathways of repair for DSBs are Homologous Recombination (HR) repair and NonHomologous End-Joining (NHEJ) repair. The HR repair requires an undamaged DNA strand as a template to repair without error the damaged cells and occurs primarily in the $S / G_{2}$ phase. Conversely, NHEJ occurs mainly during $\mathrm{G}_{1}$ phase of the cell cycle, and does not require a template and they are error prone in repair. Incorrect DNA repair can result in genetic aberrations or tumour cell death. Tumour cells that also have the capacity to repair damage induced by radiation are often radio-resistant as they typically occupy a more hypoxic environment.

Radiation induced damage can be detected/measured using a range of methodologies, for example an early cellular response to DSBs could be detected with the formation of $\gamma \mathrm{H} 2 \mathrm{AX}$ of the damaged DSBs in the nucleus (Redon et al., 2009; Mah et al., 2010; Hill, 2019) and can be used to evaluate underlying repair mechanisms (not discussed herein) and associated kinetics (Figueroa-González and Pérez-Plasencia, 2017). Common methods used to measure chromosomes aberration and mutation are the micronuclei assay; cytokinesis-block micronucleus; cytokinesis-block micronucleus cytome (CBMN) Cyt assay and many others (not discussed here). The micronuclei assay measures the chromosomal loss or breakage by the expression of micronuclei (MN) after cells have completed one nuclear division with the aid of cytochalasin B that inhibits cytoplasmic division enabling the accumulations of all dividing cells at the bi-nucleated stage. CBMN assay effectively measure the genotoxic, cytostatic and cytotoxic effects of radiation on the cells (Fenech, 2007). Moreover, it provides the proliferative index of the treated cells and their radiosensitivity (Guo et al., 1999). The viability status (apoptosis and necrosis) is shown in Fig. 2; the mitotic status (mono-nucleated, bi-nucleated cells, giant nucleated cell and multinucleated cells) in Fig. 3; and its chromosomal damage or instability status (presence of MN, nucleoplasmic bridges (NPBs), nuclear buds (NBs)), appropriate for radio-sensitivity measurements (Fenech et al., 1999), is shown in Fig. 4. 


\section{Indirectly ionizing radiation}

$\gamma$ and $x$-rays

electrons
Neutrons

Recoil protons and nuclei

\section{Directly ionizing radiation}

Ionizing particles

$[\alpha, \beta$, protons and ions (light or heavy)]

\section{Ionization or Excitation}

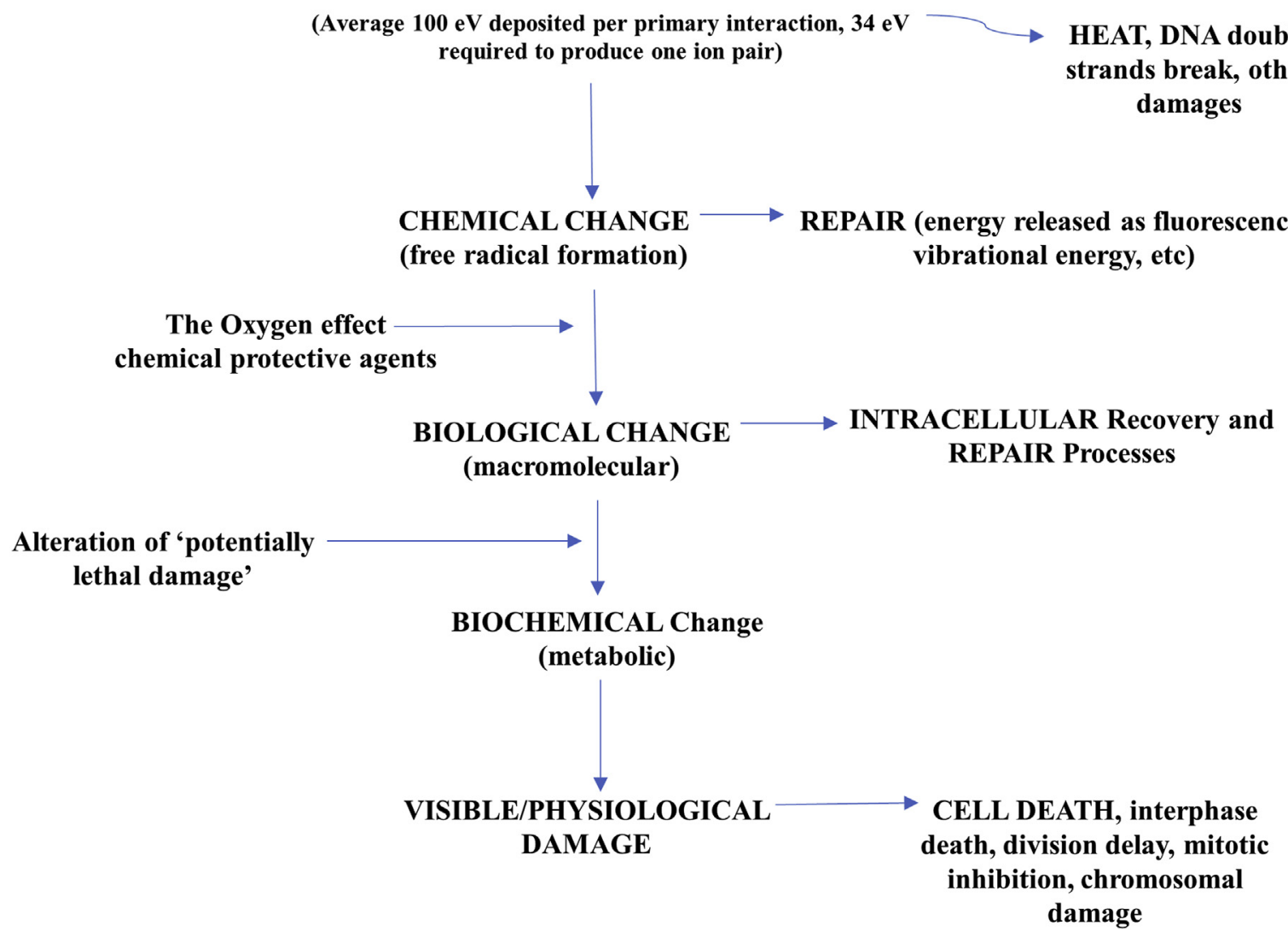

Fig. 1. Effects of radiation in cells (adapted from Little, 1968).

Briefly, MNs are induced chromosome aberration due to unrepaired or misrepair DSBs by the NHEJ repair pathway (mentioned above). NPBs are biomarkers of DNA misrepair and/or telomere end-fusion. NPBs originate from dicentric chromosomes (induced by misrepair of a chromosome break). Assessment of NPB is a form of measurement of asymmetrical chromosome rearrangement (dicentric and ring chromosome) in cells after a single division (Thomas et al., 2003). NBs are biomarkers of the elimination of over-amplified DNA and/or DNA repair complexes. This cellular process is characterized by the production of multiple copies of a particular gene or genes to amplify the

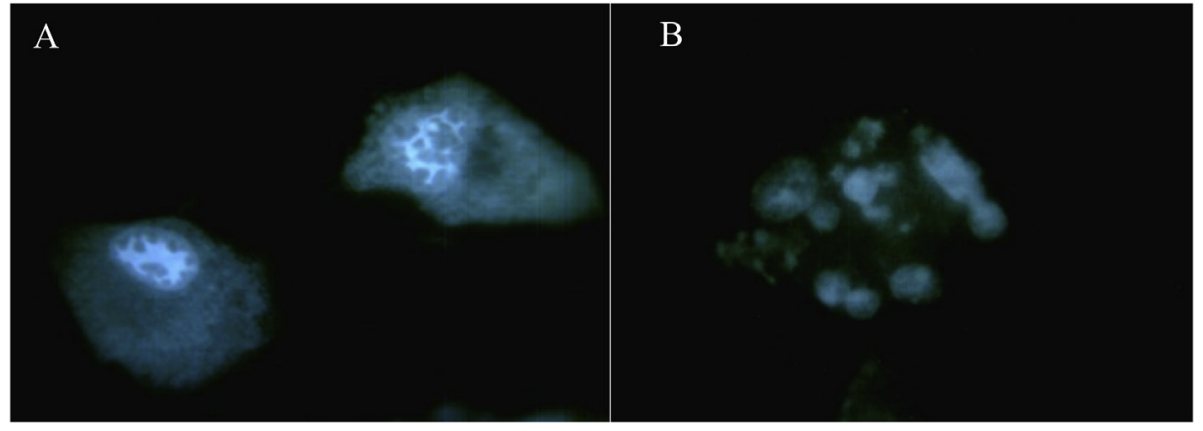

Fig. 2. Viability status: (A) two necrotic cells and (B) an apoptotic cell (x1000 magnification). 


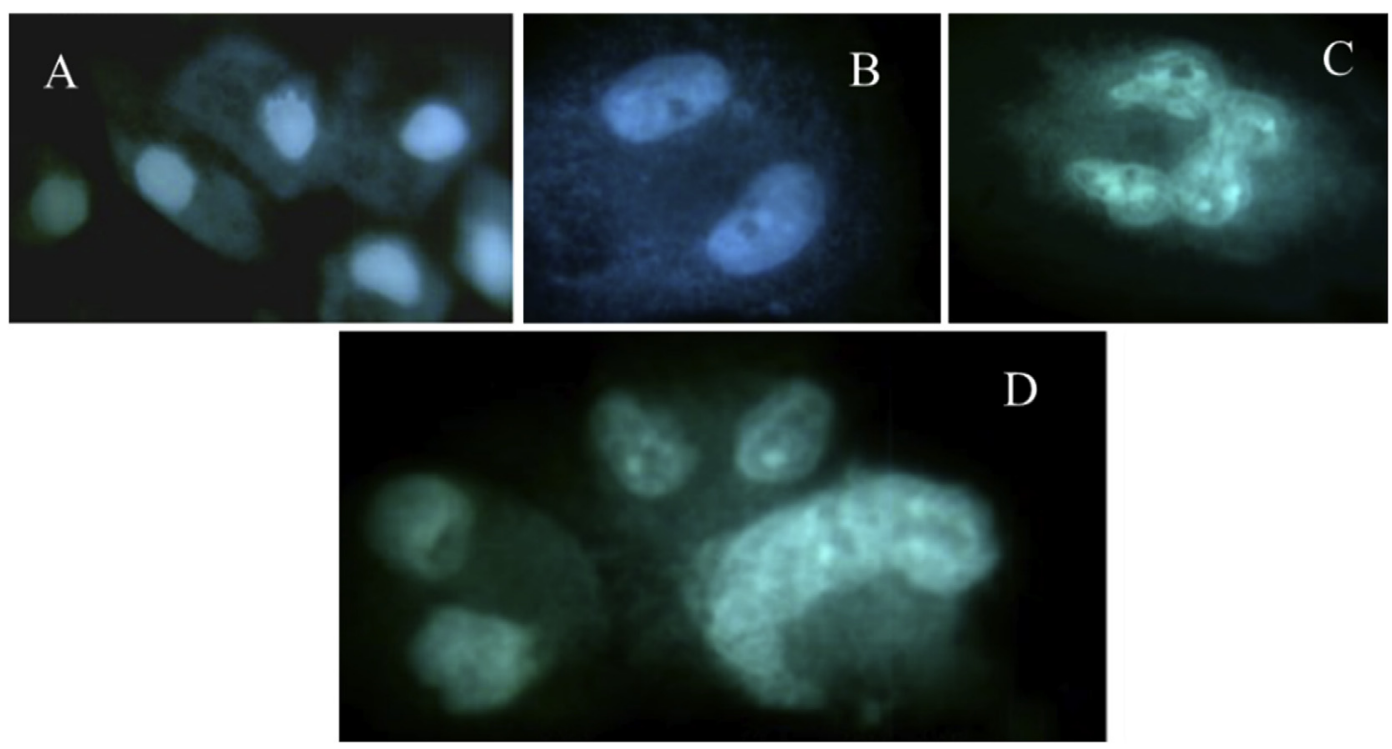

Fig. 3. Mitotic status: (A) mononuclear cells; (B) single binucleated cell; (C) multinucleated cell joined together; (D) one single giant nucleated cell (x1000 magnification).

phenotype and NBs are formed because of the capacity of the nucleus to 'sense' excess DNA that does not fit well within the nuclear matrix (nuclear housekeeping) (Fenech and Crott, 2002).

\subsection{Linear quadratic model for fractionated radiotherapy}

The Linear Quadratic (LQ) model for cell kill is commonly used in radiotherapy for quantitative predictions dose/fractionation dependencies (Jones and Dale, 2018). There are two important parameters to cell kill in the LQ formalism, embodied in the equation:

$E=\alpha d+\beta d^{2}$

Where:

E (logarithmic cell kills) is the fraction of cells killed by an absorbed dose d;

$\alpha$ is the linear component of cell kills related to DSBs caused by a single hit of radiation;

$\beta$ describes double hits (the quadratic) component of cell kills.

The $\alpha / \beta$ ratio is the dose, typically quoted in gray (Gy), when the number of cells killed by a single hit is equal to the cells killed by the quadratic components. The higher the $\alpha / \beta$ ratio, the greater the number of cells killed. Early responding tissues (jejunum) have a high $\alpha / \beta$ ratio and late responding tissues (spinal cord, brain) have a low $\alpha /$ $\beta$ ratio (see Table 1 ). The $\alpha / \beta$ ratios describe the effectiveness of the dose, often used to quantify fractionation sensitivity of tissues.

\section{Beneficial and detrimental effects (a two-edged sword)}

Sparsely ionizing radiations are limited by the 5 'Rs' of radiobiology (Steel et al., 1989), more recently the 6 'Rs', as previously mentioned. The goal of radiation is to kill all tumour cells without triggering serious damage to the normal surrounding tissues and organs at risk (OAR). To achieve this goal, fractionated radiotherapy was implemented to spare late-reacting normal tissues which have a higher repair capacity; the use of single high dose treatment was considered inefficient for other reasons (lack of elimination of hypoxic cells and exposure of cells within resistant phases of the cell cycle) and could be detrimental not only to the tumour but also to surrounding normal tissues if included in the high dose treatment volume. Fractionation, in contrast, allows tumour cells to redistribute into the mitotic phase which is radio-sensitive and it reduces hypoxia by reoxygenation (Withers, 1975).

\subsection{The 5 ' $R s$ '}

\subsubsection{Repair}

In mammalian cells, radiation induced DNA DSBs spontaneously trigger a DDR process as described above and DNA repair depends on several factors, such as types of damage, cell cycle phase of cell and repair pathways. The $S$ and $G_{2}$ phases are significant for HR while, NHEJ occurs primarily during $G_{1}$ and early $S$ phases of the cell cycle. The repair phase of damaged DNA for both tumour cells and normal cells if repaired incorrectly would contribute to chromosomal aberrations/mutation or tumour cell death. Tumour and normal tissue cells
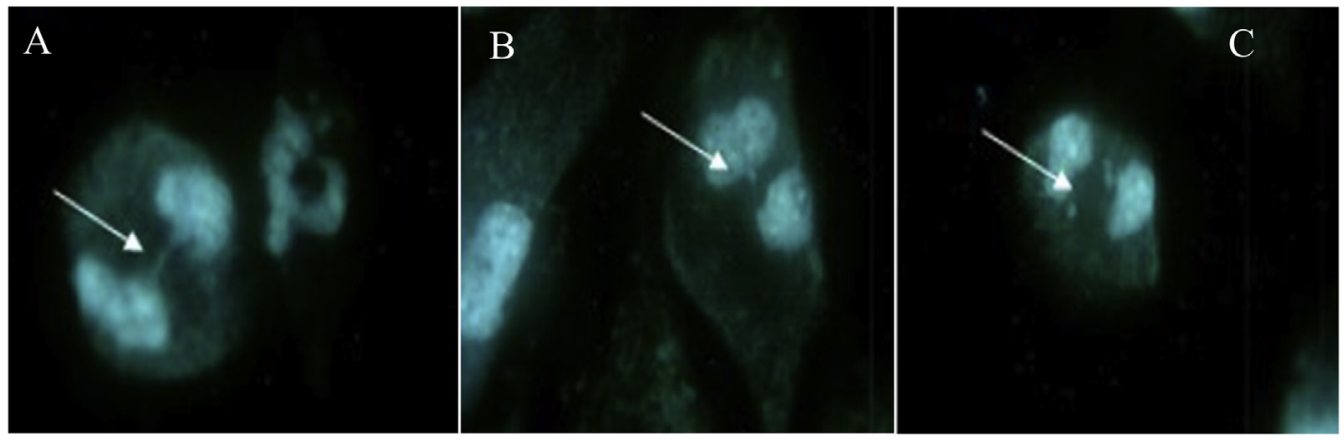

Fig. 4. Instability status: (A) nucleoplasmic bridge; (B) nuclear bud; (C) micronuclei in a binucleated cell (x 1000 magnification). 


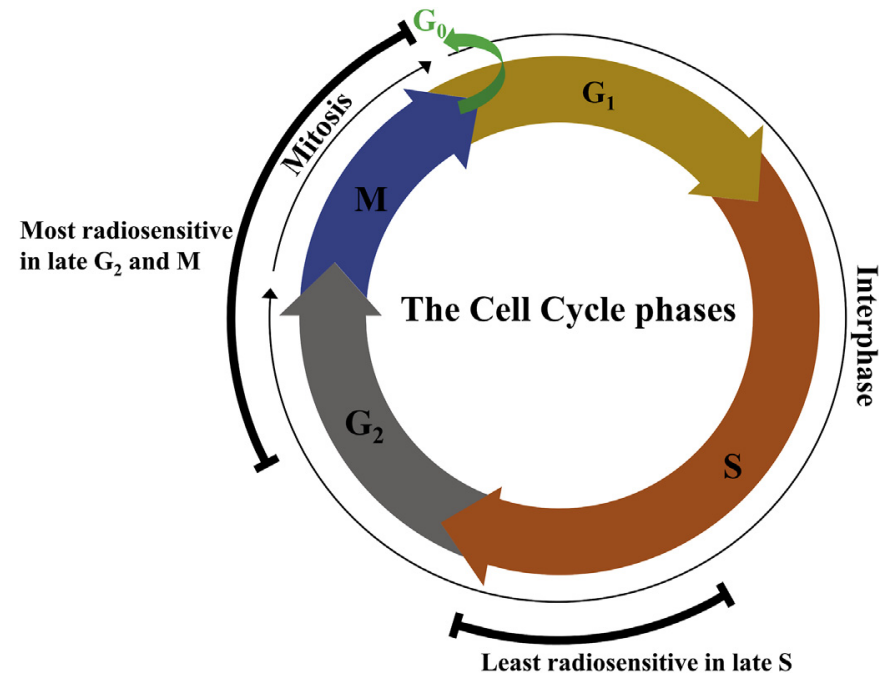

Fig. 5. Cell cycle phases with the most and least radiosensitive phases.

that have a high capacity to repair DNA damage induced by radiation are said to be radio-resistant.

Potential lethal damage plays an indirect role in affecting the radiosensitivity of cells and the radiocurability of tumours when the PLD cells are allowed to repair by arresting their proliferation (Weichselbaum et al., 1982; Weichselbaum, 1986; Weichselbaum and Beckett, 1987).

The above processes, including the repair kinetics of cells, with fast and slow repair half-time components, can be formulated mathematically and are useful in clinical decision making for optimisation purposes (Dale, 2019; Jones and Hopewell, 2018).

\subsubsection{Reassortment/redistribution}

Redistribution refers to radiation-induced cell cycle effects (Withers, 1975). The Cell Cycle is a 4-phases process comprising of Gap $1\left(G_{1}\right)$, Synthesis (S), Gap $2\left(\mathrm{G}_{2}\right)$, Mitosis and $\mathrm{G}_{0}$ (dormant phase). After completion of the cycle, the cell will either start the whole process again from $G_{1}$ or exit the cycle through $G_{0}$. The phases in the cell cycle between mitosis and the next is known as Interphase $\left(G_{1}, S\right.$ and $\left.G_{2}\right)$ as shown in Fig. 5.

To maintain genomic integrity, there is a complex network of cyclindependent kinases (CDKs) and checkpoints which prevent or delay progression of cells through the cell cycle. Both, $\mathrm{G}_{1}-\mathrm{S}$ and $\mathrm{G}_{2}-\mathrm{M}$ phases transition are under constant surveillance for protection of cells from exogenous and endogenous DNA damaging agents. Ionizing irradiation induces arrest in the $G_{1}, S$ and $G_{2}$ phases of the cell cycle.

The $G_{1}$ checkpoint is vital in initiation of cell division and is sensitive to growth factors, nutrient and other conditions to promote proliferation. Once initiated, the cell increases in size and duplicate the cellular contents. Cells that are irradiated during this phase will exhibit a delay in entering into $S$ phase. Cells irradiated at $S$ phase demonstrate a dose-dependent reduction in the rate of DNA synthesis and this increases the overall length of time to replicate their DNA substances (Joiner and Van der Kogel, 2016). S-phase cells have the opportunity to arrest and repair in the $\mathrm{G}_{2}$ phase. The DDR process is triggered to repair radiation induced DNA DSBs, a complex association between checkpoint activation and DNA repair with the involvement of proteins and related genes. There are two radiation-inducible checkpoints at $\mathrm{G}_{2}$ phase, the first checkpoint being a dose-dependent block progression of radiation damaged $\mathrm{G}_{2}$ cells to $\mathrm{G}_{1}$-or $\mathrm{S}$ - phase (Sinclair, 1968). Post radiation, the second $\mathrm{G}_{2}$ checkpoint is activated and prevent radiationdamaged $G_{2}$-phase cells from progressing through $G_{2}$ and prematurely enter mitosis with unrepaired radiation-induced DNA damage (Xu et al., 2002). Mitosis is the process of cell division involving four phases 
(Prophase, Metaphase, Anaphase and Telophase) before cytokinesis. Cytokinesis involves a division of the cytoplasm, being the process where the parent cells becomes two identical daughter cells.

Cells have different radiation sensitivities at different phases of the cell cycle; the most radiation sensitive phase is late $G_{2} / M$ phase, followed by $G_{2}, G_{1}$, early $S$, while the most radio-resistant is late $S$ phase (Tobias, 1985) as shown in Fig. 5.

Tumour cells are more sensitive in $\mathrm{G}_{2} / \mathrm{M}$ phases of the cell-cycle than $G_{1} / S$ and, when they are blocked in $G_{2} / M$ due to a functional $G_{2}$ checkpoint after exposure to radiation, they are more susceptible to the subsequent radiation ( $\mathrm{Ng}$ et al., 2013). Moreover, tumour cells have shorter cell cycle times in comparison to normal tissues. In contrast, normal cells are mostly in $G_{0} / G_{1}$ due to $G_{1}$ checkpoint and are thus less susceptible to this type of sensitisation (Ng et al., 2013). Fractionation in radiotherapy enables the surviving cells from one fraction, which will be predominantly in the radioresistant phases, to redistribute themselves into a more sensitive phase of the cell cycle to allow effective killing (therapeutic gain) for the subsequent fraction. This also favours survival of normal late responding tissues (Withers, 1975; Hall and Giaccia, 2012).

\subsubsection{Repopulation}

Repopulation also known as Regeneration where fractionation radiotherapy allows normal tissues to repopulate which is important to reduce overkill and severe side-effects for radio-sensitive tissues such as the skin or mucosa and surrounding normal tissues. For the early responding normal tissues, the fractionation interval brings about increase in radiation tolerance with increasing overall treatment time. When the interval time between two dose fraction exceeds the cell cycle, there will be an increase in the number of cells surviving due to cell proliferation. Just as normal cells can proliferate, tumour cells can also respond with an increase in rate of repopulation. At the same time as the tumour shrinks post treatment, surviving tumour cells proliferate at an accelerated rate since tumour cells have an uncontrolled growth rate, and this counteracts the cell killing effects of radiotherapy. The repopulation time of tumour cells varies during radiotherapy (Withers, 1975 ) and has a negative effect on radiation doses. Consequently, it follows that restriction of overall treatment time can be advantageous in rapidly proliferating tumour types provided that normal tissue fractionation is sufficient. An optimisation of the two processes can in principle be achieved by graphical or differential calculus methods providing that the cell and tissue parameters used are sufficiently well known (Jones and Dale, 2000).

\subsubsection{Reoxygenation}

The role of oxygen on biological response by affecting the chemical changes produced directly in the cells by radiation was first demonstrated by Gray et al. (1953). Cells irradiated under hypoxic conditions are significantly less sensitive to radiation than when oxygen is present. Following irradiation DNA radicals (DNA) as a result of indirect damage, mainly via hydroxyl radicals, can be restored (chemically repaired) to its original structure through reactions with reducing species such as thiols. However, in the presence of oxygen then this may react to produce a non-restorable organic peroxide $\left(\mathrm{DNA}-\mathrm{O}_{2}{ }^{\circ}\right.$ ) thus 'fixing' DNA into a permanent irreparable state (Hall and Giaccia, 2012; Grimes and Partridge, 2015); this cannot occur in the absence of oxygen. Oxygen effects in radiation is known as the Oxygen Enhancement Ratio (OER) which is a measure of the increase in cell sensitivity to radiation in the presence of oxygen. It is expressed as the ratio of radiation dose required to produce a given effect in the absence of oxygen to the dose required to produce the same effect in $1 \mathrm{~atm}$ of air (Hall and Giaccia, 2012). Tumours that are typically hypoxic (low oxygen level) consequently show enhanced radio-resistance to photon radiation and therefore require higher dosage of radiation to inactivate them. Reoxygenation has accounted for much of the success of fractionated radiotherapy of hypoxic tumour cells; when a radiated tumour has shrunk and re-oxygenation of tumour occurs (Withers, 1975) due to reopening of temporarily occluded blood vessels; and also, resorption of dead cells which lead to decreased distance from capillaries to tumour cells (thus improving oxygen supply). The oxygen effect is greater for low LET than high LET radiation.

Generally, it is accepted that longer duration courses of radiation allow re-oxygenation in many human tumours; but for shorter treatment courses the use of hypoxic cell sensitisation may be indicated, especially as the clinical toxicities caused by these drugs can be related to the duration of exposure.

\subsubsection{Radiosensitivity}

Different types of cell exhibit different intrinsic radio-sensitivity which can be unique to the individual (Steel et al., 1989). Radiation repair is the ability of the cells to repair sublethal damage (Elkind and Sutton, 1959; Withers, 1975) and potential lethal damage (Phillips and Tolmach, 1966). Different organs and different tissues have their own intrinsic radiosensitive property. The law of Bergonie and Tribondeau describes that tissue radiosensitivity depends on cells that are undifferentiated, have a high division/proliferation capacity and divide rapidly (Bergonie and Tribondeau, 1959) except lymphocytes; Casarett's Classification according to tissue organization, cell kinetics and microcirculation (Rubin and Casarett, 1968); and tissue architecture according to Michalowski's Classification (Wheldon et al., 1982). That implies that cells that divide and regenerate slowly are the most resistant. Conversely, there are some glioblastoma cell lines that are lowdose hypersensitive to radiation (Short et al., 1999).

In general, radiosensitivity is inversely related to repair capacity. Thus, highly radiosensitive cells have low DNA repair capacity reflected in their high $\alpha / \beta$ ratios which reflect the ratio of $\alpha$, the coefficient of cell kill per unit dose and $\beta$, the coefficient of cell kill per unit dose squared. Radioresistant cells have low $\alpha / \beta$ ratios. Larger doses per treatment are often suggested for low $\alpha / \beta$ systems and small doses per fraction for high $\alpha / \beta$ systems (Jones and Dale, 2018). A summary of the 5 'Rs' dose rate and LET effects on fractionated radiotherapy is provided in Table 1.

\subsection{Other phenomena of radiations as included in Table 2}

\subsubsection{Bystander effects}

The "Bystander effects" refer to the induction of biological effects in cells that are not directly traversed by radiation. The non-irradiated cells that are located nearby the irradiated cells have also been observed to respond to exposure, including the induction of DNA damage and a reduction in clonogenic survival. This implies the target for radiation biological effects is larger than the cell, observed in both highand low- LET radiations (Nagasawa and Little, 1999). The bystander effect was demonstrated when Nagasawa and Little (1999) irradiated less than $1 \%$ of cell nuclei with low doses of $\alpha$ particles, with an unexpected high frequency of sister chromatid exchanges (Nagasawa and Little, 1999). A wide range of bystander effects have been observed including reduced clonogenic survival, increased sister chromatid exchanges, formation of micronuclei and apoptosis, genomic instability and chromosomes abnormalities (Nagasawa and Little, 1999); apoptosis, cell death (Koturbash et al., 2008) and all these effects are similar to the direct effect of radiation. Azzam et al. (1998) demonstrated that the changes occur via cellular communication (Azzam et al., 1998; Zhou et al., 2005), when irradiated cells send damage signals through gap junctions to the neighbouring non-targeted cells and act as though they have been hit by radiation (Azzam et al., 1998; Mothersill and Seymour, 2001; Seymour and Mothersill, 2004; Prise and O'Sullivan, 2009). Cytokine (Kaminski et al., 2005), signalling pathways (Lehnert and Goodwin, 1997; Narayanan et al., 1997; Iyer, 2000) and other mechanisms such as inflammation, immune system, gene expression and epigenetic modulators (Ilnytskyy and Kovalchuk, 2011), including extracellular vesicles/exosomes (Jabbari et al., 2020; Le et al., 2017), 
Table 2

Other less dominant phenomena of radiotherapy.

\begin{tabular}{|c|c|c|}
\hline Phenomenon & Features & Authors \\
\hline Bystander effect & $\begin{array}{l}\text { Neighbour non-irradiated cells exhibit radiation induced DNA damage and cellular consequences } \\
\text { similar to the irradiated cells, mediated by cytokine, cell-signalling through gap junctions and } \\
\text { inflammatory responses. Involves abscopal effect and secondary cancers. Most effective with } \\
\text { radiation doses of }<1 \mathrm{~Gy} \text { but is also applicable to doses }>10 \mathrm{~Gy} \text {. }\end{array}$ & $\begin{array}{l}\text { (Prise and O'Sullivan, 2009; Hamada } \\
\text { et al., 2011) }\end{array}$ \\
\hline Low dose hyper-radiosensitivity & $\begin{array}{l}\text { Usually for doses }<0.3 \mathrm{~Gy} \text {, in rapidly proliferating cells with a high } \mathrm{G}_{2} \text { cell cycle component. The } \\
\text { initial cell survival curve is linear and steep followed by shallower curve. Thought to be due to } \\
\text { induced repair (failure to arrest in } \mathrm{G}_{2} \text { ). Cells become radioresistant at higher doses. Low doses } \\
\text { fractionated radiation therapy could be recommended. }\end{array}$ & $\begin{array}{l}\text { (Joiner et al., 2001; Short et al., 2001; } \\
\text { Marples et al., 2004) }\end{array}$ \\
\hline Adaptive response (AR) & $\begin{array}{l}\text { AR is an active process where low dose exposure induces 'tolerance' to subsequent high dose } \\
\text { exposure. The low-dose irradiated cells are stimulated to react and become more resistant to } \\
\text { subsequent irradiation (biological defence mechanism). }\end{array}$ & $\begin{array}{l}\text { (Olivieri et al., 1984; Wolff, 1998; } \\
\text { Hamada et al., 2011) }\end{array}$ \\
\hline Hormesis & $\begin{array}{l}\text { Low dose radiation for health. Beneficial at low doses but toxic at high doses. Low dose beneficial } \\
\text { effect stimulates the immune system and adaptive responses improve the functional ability of cells } \\
\text { and the organism. The physiologic response to ionizing radiation is directly proportional to the } \\
\text { logarithm of the dose. }\end{array}$ & $\begin{array}{l}\text { (Luckey, 2006; Calabrese, 2014; } \\
\text { Calabrese and Mattsonm, 2017) }\end{array}$ \\
\hline Abscopal effect & $\begin{array}{l}\text { Indirect anticancer effects of radiotherapy on tumours out of the radiation field. Local irradiation of } \\
\text { one tumour tissue involved in a response of another or similar tissue away from the irradiated site. } \\
\text { This systemic effect of the irradiated tumours is mediated by radiation-induced cytokines, relevant } \\
\text { factors causing a chronic inflammatory environment, leading to induction of genomic instability } \\
\text { and radiation susceptibility in both distant tumour (regression) and normal tissue (secondary } \\
\text { carcinogenesis). }\end{array}$ & (Mole, 1953; Siva et al., 2013) \\
\hline
\end{tabular}

also contribute to the bystander effects. These effects are inherently linked to perturbation of intercellular signalling, which not only results in effects observed in non-irradiated cells, but can also modify the response of surrounding irradiated cells. Such effects have been demonstrated across large dose gradients, similar to that produced by clinical beams, where the experimental data show a modification of cell survival in the beam as a result of bystander signals effects.

This two-edged sword of bystander-effects, having anti-tumour abscopal effects (see below) and abscopal effects in normal tissues, would certainly seem to require attention in the radiotherapy treatment of patients. As an example, normal oxygenated tissues will experience greater cell death due to bystander-related mechanisms (Marín et al., 2014); the bystander effects are one of the complications in radiation exposure, potentially causing chromosomal damages that could lead to near future carcinogenic risk (Dent et al., 2000). Conversely, bystandereffects signalling (McMahon et al., 2015), autoimmune diseases or chronic inflammation may also be beneficial to radiotherapy (Schaue, 2017). Thus, modification of bystander signalling could in principle be used to modify radiation responses, but this does not appear to have been realised so far. It is possible that the effect is an in vitro artefact, similar to reduced PLD repair in vitro.

\subsubsection{Low dose hypersensitivity}

Low dose hypersensitivity (HRS) is the phenomenon in which irradiated mammalian cells are sensitive to doses below $\sim 0.5$ Gy but become increased in radioresistance with higher doses, especially in regard to radioresistant cells (Joiner et al., 1996). These HRS cells are sensitive to low single doses as these low damage levels do not trigger the cells to repair. With higher doses, HRS is followed by an increase in radioresistance (Wouters et al., 1996). The mechanisms of HRS involve; hypersensitivity of a subpopulation of the cells, impaired cell-cycle regulation and a defective DNA repair system (Short et al., 2003, 2005; Marples and Collis, 2008). Moreover, HRS could be a protective mechanism that has evolved to prevent cells from developing genomic instability and mutations (Marples and Collis, 2008; Martin et al., 2014).

\subsubsection{Adaptive response}

Adaptive response refers to cells that are pre-exposed to very low doses of ionizing radiation and with subsequent exposure to high dose will result in less genetic damage in the pre-exposed cells compared to those that were not pre-exposed. This is attributed to the induction of repair mechanisms triggered by the low dose exposure. This implies that exposure of cells to a low dose of ionizing radiation can induce a condition of enhanced radioresistance termed as "radio-adaptive response" (Mothersill, 2006; Azzam and Little, 2004). Currently there is very little information of the potential impact (if any) of this effect on radiotherapy and how it could potentially be utilised to improve therapeutic outcome.

\subsubsection{Hormesis}

Radiation hormesis is a biphasic dose response phenomenon in which adaptive responses to low doses of ionizing radiation are beneficial but are harmful at high doses (Luckey, 2006). The immune system response to an 'adapting' or 'pre-conditioning' low-dose tends to elicit stress responses and stimulate adaptation, a protective mechanism (Feinendegen, 2005). This is contrary to the Linear-No-Threshold (LNT) concept. Hormesis implies no exposure and very high-dose exposures suppress the immune system as compared to low doses. The LNT model of radiation risk is used for radiation protection purposes but has overlooked the adaptive defences of the body that are triggered by low dose radiation. This LNT-hypothesis has been described as inconsistent by numerous experiments, as shown by both laboratory experiments and in human exposures (Feinendegen et al., 2012; Oakley, 2015).

\subsubsection{Abscopal effect}

Abscopal (out of field) effects are related to bystander effects but specifically refer to responses of other organs and tissues at a distance from the target tissues (Mole, 1953). Murphy and Morton (1915) removed tumours from mice, irradiated the mice, and returned the tumours to the mice; comparison was made to the control group with the tumours intact during irradiation (Murphy and Morton, 1915). The results observed was that unirradiated tumours grafts shrank by $50 \%$ and survived for approximately five weeks, while the control group members died after one week, although these results could be due to vascular disruption. Abscopal effects can also include induction of genomic instability (Lorimore et al., 2003), cell death, and oncogenic transformation in normal tissues. This out of field effect of localized irradiation perturbing the organism as a whole, could be either beneficial or detrimental (Siva et al., 2013). This two-edged sword, with anti-tumour abscopal effects and abscopal effects in normal tissues also require attention in the radiotherapy treatment for patients, in particular there being very little information on how these effects are modulated by fractionations. 


\subsection{Current trend of radiotherapy - Particle radiotherapy}

As presented above, where several of the 5 "Rs" constrain the effectiveness of photon radiotherapy for tumours, an alternative treatment modality such as charged particle radiotherapy would seems to be more effective, albeit, not for all types of tumours. Charged particle therapies such as the use of proton and carbon ions have several characteristics that are attractive to effective radiotherapy such as; high LET resulting in higher relative biological effectiveness (RBE), reduced repair capacity, lower OER and decreased cell-cycle dependence that are less affected by the 5 "Rs" of radiobiology. Increasing LET results in an increase in the frequency and complexity of these complex DSBs which are more difficult to repair than simple DSBs, leading to an increase in RBE. In addition to simple DSB, ionizing radiation can also induce complex DSB, which are comprised of a DSB with additional strand breaks and/or base damage within a few base pairs (Ward, 1985; Goodhead, 1994, 1999). For high LET radiation, even in the absence of oxygen, the complexity of the induced DNA damage is such that they are very difficult to repair,altering the level of oxygen having little effect on the resulting biological response (Goodhead, 1994, 1999). Likewise, the response is more or less independent of dose-rate, but where lower LET values are found these mechanisms do modulate the response. Therefore, as LET increases, RBE increases and OER decreases. Hence, proton radiotherapy OER will be higher than carbon ion radiotherapy.

RBE compares the efficiency of different types of radiations to produce a defined biological effectiveness referenced to photon radiation. RBE is dependent on many factors such as LET, cell and tissue type, biological endpoints, dose, dose rate or fractionation, ion species, oxygenation status and many other influences (Hall and Giaccia, 2012; Held et al., 2016). Clinically, RBE is used to calculate the Gy Equivalent dose (GyE), the physical doses being multiplied by the RBE of the related ion used. The RBE for protons is low at $\sim 1.2$, similar to high energy X-rays (Paganetti, 2002) and for carbon ions is $\sim 3$ for treatment (Matsufuji et al., 2003; Tsujii et al., 2007). Although, proton and carbon ion radiotherapy have superior dose distribution, the principal difference is LET. Protons have low LET and carbon ions have higher LET.

Moreover, the Bragg peak of particle also provides excellent precision at targeting of tumours by deposition maximum energy at the tumour and minimising dose to OAR (Wilson, 1946). Larger tumours can be covered by using particles of different energies to produce a spreadout Bragg peak which can encompass the tumour accurately with precise imaging.

Owing to each of the heavy ion properties, there are always physical limitations and uncertainties such as 'delta rays' $(\delta)$ arising from the initial ionization events, multiple scattering from Coulomb interactions with nuclei similar to the interactions with electrons, and range straggling due to energy loss through interactions of ions with orbiting electrons, losing a varying amount of energy with each interaction (Chu, 2006; Lomax, 2009). These ion beam effects vary approximately with the inverse square-root of the mass of the particle and could be minimised by removing material from the beam line. Moreover, nuclear fragmentations especially from heavier ions such as carbon ions also degrade the accuracy of treatment planning and could undermine the primary benefits of heavy ion radiotherapy as it contributes significantly to the dose within the spread-out Bragg peak (Chu, 2006). All these uncertainties and limitations, related to charged ion radiation (delivery, treatment planning, physical and biological tissues variabilities and doses) have been considered, studied and greatly overcome in advance of the start of treatment and are continuously being improved (Kanai et al., 1999; Matsufuji et al., 2003; Jäkel, 2009; Suit et al., 2010).

\section{Conclusion}

Radiotherapy is in principle a local treatment modality that is harnessed towards combating cancer. However as in present discussions and with clinical evidence of long-range beneficial and other effects, the issue of needing well-controlled radiation treatment is obvious. Cells, tissues and organ response to radiation is multifactorial and complex in nature. With increasing use of small-field (few $\mathrm{mm}$ and less), high-dose per-fraction (dose escalation) treatments and high LET radiotherapy, increasing consideration also needs to be made of the nature of the human body response. A novel systemic mediator that could control tumour expansion and also defend normal tissue would be most desired. The ability to be able to predict the potential consequences of offtarget effects would enhance the radiation effectiveness and radiation risk assessment. Moreover, out of field effects could also be factored into treatment planning. Additionally, there are exciting prospects for radiotherapy where new targets for adjuvant therapy are now possible. It is also necessary to use mathematical optimisation techniques within models of radiotherapy to combine the often-qualitative biological knowledge with the quantitative effects of radiation provided that the key operative parameters are known. Currently, the benefits for extending patients' lives with quality of life would outweigh the undesirable body responses to radiation treatment.

\section{Declaration of competing interest}

The authors declare that they have no known competing financial interests or personal relationships that could have appeared to influence the work reported in this paper.

\section{Acknowledgement}

We would like to thank Sunway University for the internal grant (INT-2019 SHMS-CBP-03).

\section{Appendix A. Supplementary data}

Supplementary data to this article can be found online at https:// doi.org/10.1016/j.radphyschem.2020.108994.

\section{References}

Azzam, E.I., de Toledo, S.M., Gooding, T., Little, J.B., 1998. Intercellular communication is involved in the Bystander regulation of gene expression in human cells exposed to very low fluences of alpha particles. Radiat. Res. 150, 497-504.

Azzam, E.I., Little, J.B., 2004. The radiation-induced bystander effect: evidence and significance. Hum. Exp. Toxicol. 23, 61-65.

Bergonie, J., Tribondeau, L., 1959. Interpretation of some results of radiotherapy and an attempt at determining a logical technique of treatment/de quelques resultats de la radiotherapie et Essai de Fixation d'une Technique Rationnelle. Radiat. Res. 11, $587-588$.

Calabrese, E.J., 2014. Hormesis: a fundamental concept in biology. Microbial cell (Graz, Austria) 1, 145-149.

Calabrese, E.J., Mattsonm, M.P., 2017. How does hormesis impact biology, toxicology, and medicine? NPJ Aging Mech Dis 3, 13.

Chu, W.T., 2006. Overview of Light-Ion Beam Therapy. IAEA-TECDOC-1560.

Dale, R.G., 2019. Radiation repair models for clinical application. Br. J. Radiol. 92, e20180070.

Dent, S.F., Klaassen, D., Pater, J.L., Zee, B., Whitehead, M., 2000. Second primary malignancies following the treatment of early stage ovarian cancer: update of a study by the National Cancer Institute of Canada_clinical Trials Group (NCIC-CTG). Ann. Oncol. 11, 65-68.

Elkind, M.M., Sutton, H., 1959. X-ray damage and recovery in mammalian cells in culture. Nature 184, 1293-1295.

Feinendegen, L.E., 2005. Evidence for beneficial low level radiation effects and radiation hormesis. Br. J. Radiol. 78, 3-7.

Feinendegen, L., Pollycove, M., Neumann, R., 2012. Hormesis by Low Dose Radiation Effects: Low-Dose Cancer Risk Modeling Must Recognize Up-Regulation of Protection. Therapeutic Nuclear Medicine. Springer, pp. 789-805.

Fenech, M., Crott, J., Turner, J., Brown, S., 1999. Necrosis, apoptosis, cytostasis and DNA damage in human lymphocytes measured simultaneously within the cytokinesisblock micronucleus assay: description of the method and results for hydrogen peroxide. Mutagenesis 14, 605-612.

Fenech, M., Crott, J.W., 2002. Micronuclei, nucleoplasmic bridges and nuclear buds induced in folic acid deficient human lymphocytes-evidence for breakage-fusionbridge cycles in the cytokinesis-block micronucleus assay. Mutat Res-Fund Mol M. 
504, 131-136.

Fenech, M., 2007. Cytokinesis-block micronucleus cytome assay. Nat. Protoc. 2, 1084-1104.

Figueroa-González, G., Pérez-Plasencia, C., 2017. Strategies for the evaluation of DNA damage and repair mechanisms in cancer. Oncol Lett 13, 3982-3988.

Goodhead, D.T., 1994. Initial events in the cellular effects of ionizing radiations : clustered damage in DNA. In: Radiat Res. Annual Meeting. Taylor \& Francis, London, pp. 7-17 Guilford 1993-07-12.

Goodhead, D.T., 1999. Mechanisms for the biological effectiveness of high-LET radiations. J. Radiat. Res. 40, S1-S13.

Gray, L.H., Conger, A.D., Ebert, M., Hornsey, S., Scott, O.C.A., 1953. The concentration of oxygen dissolved in tissues at the time of irradiation as a factor in radiotherapy. Br. J. Radiol. 26, 638-648.

Grimes, D.R., Partridge, M., 2015. A mechanistic investigation of the oxygen fixation hypothesis and oxygen enhancement ratio. Biomed Phys Eng Express 4, e045209.

Guo, G.Z., Sasai, K., Oya, N., Shibata, T., Shibuya, K., Hiraoka, M., 1999. A significant correlation between clonogenic radiosensitivity and the simultaneous assessment of micronucleus and apoptotic cell frequencies. Int. J. Radiat. Biol. 75, 857-864.

Hall, E.J., Giaccia, A.J., 2012. Radiobiology for the Radiologist, seventh ed. Lippincott Williams \& Wilkins.

Hall, E.J., Kraljevic, U., 1976. Repair of potentially lethal radiation damage: comparison of neutron and X-ray RBE and Implications for radiation therapy. Radiology 121 731-735.

Hamada, N., Maeda, M., Otsuka, K., Tomita, M., 2011. Signaling pathways underpinning the manifestations of ionizing radiation-induced bystander effects. Curr. Mol. Pharmacol. 4, 79-95.

Held, K.D., Kawamura, H., Kaminuma, T., Paz, A.E.S., Yoshida, Y., Liu, Q., Willers, H., Takahashi, A., 2016. Effects of charged particles on human tumor cells. Front Oncol 6, 23.

Hill, M., 2019. Radiation track structure: how the spatial distribution of energy deposition drives biological response. Clin. Oncol. 32, 75-83.

Ilnytskyy, Y., Kovalchuk, O., 2011. Non-targeted radiation effects-An epigenetic connection. Mutat. Res. 714, 113-125.

Iyer, R., 2000. Factors underlying the cell growth-related bystander responses to alpha particles. Canc. Res. 60, 1290-1298.

Jabbari, N., Karimipour, M., Khaksar, M., Akbariazar, E., Heidarzadeh, M., Mojarad, B. Aftab, H., Rahbarghazi, R., Rezaie, J., 2020. Tumor-derived extracellular vesicles: insights into bystander effects of exosomes after irradiation. Laser Med. Sci. 35, 531-545.

Jäkel, O., 2009. Medical physics aspects of particle therapy. Radiat. Protect. Dosim. 137, $156-166$.

Joiner, M.C., Lambin, P., Malaise, E.P., Robson, T., Arrand, J.E., Skov, K.A., Marples, B., 1996. Hypersensitivity to very-low single radiation doses: its relationship to the adaptive response and induced radioresistance. Mutat Res-Fund Mol M. 358, 171-183.

Joiner, M.C., Marples, B., Lambin, P., Short, S.C., Turesson, I., 2001. Low-dose hypersensitivity: current status and possible mechanisms. Int. J. Radiat. Oncol. Biol. Phys. 49, 379-389.

Joiner, M.C., Van der Kogel, A., 2016. Basic Clinical Radiobiology. CRC Press.

Jones, B., Dale, R.G., 2000. Radiobiological modeling and clinical trials. Int. J. Radiat Oncol. Biol. Phys. 48, 259-265.

Jones, B., Dale, R.G., 2018. The evolution of practical radiobiological modelling. Br. J. Radiol. 92, e20180097.

Jones, B., Hopewell, J.W., 2018. Modelling the influence of treatment time on the biological effectiveness of single radiosurgery treatments: derivation of "protective" dose modification factors. Br. J. Radiol. 92, e20180111.

Kaminski, J.M., Shinohara, E., Summers, J.B., Niermann, K.J., Morimoto, A., Brousal, J., 2005. The controversial abscopal effect. Canc. Treat Rev. 31, 59-72.

Kanai, T., Endo, M., Minohara, S., Miyahara, N., Koyama-ito, H., Tomura, H., Matsufuji, N., Futami, Y., Fukumura, A., Hiraoka, T., Furusawa, Y., Ando, K., Suzuki, M., Soga, F., Kawachi, K., 1999. Biophysical characteristics of HIMAC clinical irradiation system for heavy-ion radiation therapy. Int. J. Radiat. Oncol. Biol. Phys. 44, 201-210.

Koturbash, I., Loree, J., Kutanzi, K., Koganow, C., Pogribny, I., Kovalchuk, O., 2008. In vivo bystander effect: cranial $\mathrm{x}$-irradiation leads to elevated DNA damage, altered cellular proliferation and apoptosis, and increased p53 levels in shielded spleen. Int. J. Radiat. Oncol. Biol. Phys. 70, 554-562.

Le, M., Fernandez-Palomo, C., McNeill, F.E., Seymour, C.E., Rainbow, A.J., Mothersill, C.E., 2017. Exosomes are released by bystander cells exposed to radiation-induced biophoton signals: reconciling the mechanisms mediating the bystander effect. PloS One 12, e0173685.

Lehnert, B.E., Goodwin, E.H., 1997. A new mechanism for DNA alterations induced by alpha particles such as those emitted by radon and radon progeny. Environ. Health Perspect. 105 (Suppl. 5), 1095-1101.

Little, J.B., 1968. Cellular effects of ionizing radiation. N. Engl. J. Med. 278, 369-376.

Lomax, A.J., 2009. Charged particle therapy: the physics of interaction. J. Canc. 15, 285-291.

Lorimore, S.A., Coates, P.J., Wright, E.G., 2003. Radiation-induced genomic instability and bystander effects: inter-related nontargeted effects of exposure to ionizing radiation. Oncogene 22, 7058-7069.

Luckey, T.D., 2006. Radiation Hormesis: the Good, the Bad, and the Ugly, vol. 4. Doseresponse : a publication of International Hormesis Society, pp. 169-190.

Mah, L.J., El-Osta, A., Karagiannis, T.C., 2010. $\gamma$ H2AX: a sensitive molecular marker of DNA damage and repair. Leukemia 24, 679-686.

Marín, A., Martín, M., Liñán, O., Alvarenga, F., López, M., Fernández, L., Büchser, D., Cerezo, L., 2014. Bystander effects and radiotherapy. Rep. Practical Oncol. Radiother. 20, 12-21.
Marples, B., Wouters, B., Collis, S., Chalmers, A., Joiner, M.C., 2004. Low-dose hyperradiosensitivity: a consequence of ineffective cell cycle arrest of radiation-damaged G2-phase cells. Radiat. Res. 161, 247-255.

Marples, B., Collis, S.J., 2008. Low-dose hyper-radiosensitivity: past, present, and future. Int. J. Radiat. Oncol. Biol. Phys. 70, 1310-1318.

Martin, L.M., Marples, B., Lynch, T.H., Hollywood, D., Marignol, L., 2014. Exposure to low dose ionising radiation: molecular and clinical consequences. Canc. Lett. 349, 98-106.

Matsufuji, N., Fukumura, A., Komori, M., Kanai, T., Kohno, T., 2003. Influence of fragment reaction of relativistic heavy charged particles on heavy-ion radiotherapy. Phys. Med. Biol. 48, 1605-1623.

Matsuya, Y., McMahon, S.J., Tsutsumi, K., Sasaki, K., Okuyama, G., Yoshii, Y., Mori, R., Oikawa, J., Prise, K.M., Date, H., 2018. Investigation of dose-rate effects and cellcycle distribution under protracted exposure to ionizing radiation for various doserates. Sci. Rep. 8, 8287.

McMahon, S.J., McGarry, C.K., Butterworth, K.T., Jain, S., O'Sullivan, J.M., Hounsell, A.R., Prise, K.M., 2015. Cellular signalling effects in high precision radiotherapy. Phys. Med. Biol. 60, 4551-4564.

Mole, R.H., 1953. Whole body irradiation-radiobiology or medicine? Br. J. Radiol. 26, 234-241.

Mothersill, C., Seymour, C., 2001. Radiation-induced bystander effects: past history and future direction. Radiat. Res. 155, 759-767.

Mothersill, C., 2006. Radiation-induced bystander effects and the DNA paradigm: an "Out of field" perspective. Mutat. Res. 597, 5-10.

Murphy, J.B., Morton, J.J., 1915. The effect of Roentgen rays on the rate of growth of spontaneous tumours in mice. J. Exp. Med. 22, 800-803.

Nagasawa, H., Little, J.B., 1999. Unexpected sensitivity to the induction of mutations by very low doses of alpha-particle radiation:evidence for a bystander effect. Radiat. Res. 152, 552-557.

Narayanan, P.K., Goodwin, E.H., Lehnert, B.E., 1997. a particles initiate biological production of superoxide anions and hydrogen peroxide in human cells. Canc. Res. 57, 3963-3971.

Ng, W.L., Huang, Q., Liu, X.J., Zimmerman, M., Li, F., Li, C.Y., 2013. Molecular mechanisms involved in tumor repopulation after radiotherapy. Transl. Cancer Res. 2, $442-448$.

Oakley, P., 2015. Is use of radiation hormesis the missing link to a better cancer treat ment? J. Canc. Ther. 6, 601-605.

Olivieri, G., Bodycote, J., Wolff, S., 1984. Adaptive response of human lymphocytes to low concentrations of radioactive thymidine. Science 223, 594-597.

Paganetti, H., 2002. Relative biological effectiveness (RBE) values for proton beam therapy. Int. J. Radiat. Oncol. Biol. Phys. 53, 407-421.

Phillips, R.A., Tolmach, L.J., 1966. Repair of potentially lethal damage in X-irradiated HeLa cells. Radiat. Res. 29, 413-432.

Pouget, J.P., Georgakilas, A.G., Ravanat, J.L., 2018. Targeted and off-rarget (bystander and abscopal) effects of radiation therapy: redox mechanisms and risk/benefit analysis. Antioxidants Redox Signal. 29, 1447-1487.

Prise, K.M., O'Sullivan, J.M., 2009. Radiation-induced bystander signalling in cancer therapy. Nat. Rev. Canc. 9, 351-360.

Redon, C.E., Dickey, J.S., Bonner, W.M., Sedelnikova, O.A., 2009. $\gamma$-H2AX as a biomarker of DNA damage induced by ionizing radiation in human peripheral blood lymphocytes and artificial skin. Adv. Space Res. 43, 1171-1178.

Rubin, P., Casarett, G.W., 1968. Clinical radiation pathology as applied to curative radiotherapy. Cancer 22, 767-778.

Schaue, D., 2017. A century of radiation therapy and adaptive immunity. Front. Immunol. 8, e431.

Seymour, C.B., Mothersill, C., 2004. Radiation-induced bystander effects-implications for cancer. Nat. Rev. Canc. 4, 158-164.

Short, S., Mayes, C., Woodcock, M., Joiner, M.C., 1999. Low-dose hypersensitivity in the T98G glioblastoma cell line. Int. J. Radiat. Biol. 75, 847-855.

Short, S., Kelly, J., Mayes, C., Woodcock, M., Joiner, M., 2001. Low-dose hypersensitivity after fractionated low-dose irradiation in vitro. Int. J. Radiat. Biol. 77, 655-664.

Short, S., Woodcock, M., Marples, B., Joiner, M., 2003. Effects of cell cycle phase on lowdose hyper-radiosensitivity. Int. J. Radiat. Biol. 79, 99-105.

Short, S., Bourne, S., Martindale, C., Woodcock, M., Jackson, S.P., 2005. DNA damage responses at low radiation doses. Radiat. Res. 164, 292-302.

Sinclair, W.K., 1968. Cyclic X-ray responses in mammalian cells in vitro. Radiat. Res. 33, 620-643.

Siva, S., Macmanus, M., Martin, R., Martin, O., 2013. Abscopal effects of radiation therapy: a clinical review for the radiobiologist. Canc. Lett. 356, 82-90.

Steel, G.G., McMillan, T.J., Peacock, J.H., 1989. The 5Rs of radiobiology. Int. J. Radiat. Biol. 56, 1045-1048.

Suit, H., DeLaney, T., Goldberg, S., Paganetti, H., Clasie, B., Gerweck, L., Niemierko, A., Hall, E., Flanz, J., Hallman, J., Trofimov, A., 2010. Proton vs carbon ion beams in the definitive radiation treatment of cancer patients. Radiother. Oncol. 95, 3-22.

Thomas, P., Umegaki, K., Fenech, M., 2003. Nucleoplasmic bridges are a sensitive measure of chromosome rearrangement in the cytokinesis-block micronucleus assay. Mutagenesis 18, 187-194.

Tobias, C.A., 1985. The future of heavy-ion science in biology and medicine. Radiat. Res. $103,1-33$.

Tsujii, H., Mizoe, J., Kamada, T., Baba, M., Tsuji, H., Kato, H., Kato, S., Yamada, S. Yasuda, S., Ohno, T., Yanagi, T., Imai, R., Kagei, K., Kato, H., Hara, R., Hasegawa, A., Nakajima, M., Sugane, N., Tamaki, N., Takagi, R., Kandatsu, S., Yoshikawa, K., Kishimoto, R., Miyamoto, T., 2007. Clinical results of carbon ion radiotherapy at NIRS. J. Radiat. Res. 48 (Suppl. A), A1-A13.

Ward, J.F., 1985. Biochemistry of DNA lesions. Radiat. Res. 104, S103-S111.

Weichselbaum, R.R., Schmit, A., Little, J.B., 1982. Cellular repair factors influencing 
radiocurability of human malignant tumours. Br. J. Canc. 45, 10-16.

Weichselbaum, R.R., 1986. Radioresistant and repair proficient cells may determine radiocurability in human tumors. Int. J. Radiat. Oncol. Biol. Phys. 12, 637-639.

Weichselbaum, R.R., Beckett, M., 1987. The maximum recovery potential of human tumor cells may predict clinical outcome in radiotherapy. Int. J. Radiat. Oncol. Biol. Phys. 13, 709-713.

Wheldon, T.E., Michalowski, A.S., Kirk, J., 1982. The effect of irradiation on function in self-renewing normal tissues with differing proliferative organisation. Br. J. Radiol. 55, 759-766.

Wilson, R.R., 1946. Radiological use of fast protons. Radiology 47, 487-491.

Withers, H.R., 1975. The four R's of radiotherapy. Adv. Radiat. Biol. 5, 241-271.
Wolff, S., 1998. The adaptive response in radiobiology: evolving insights and implications. Environ. Health Perspect. 106 (Suppl. 1), 277-283 1.

Wouters, B.G., Sy, A.M., Skarsgard, L.D., 1996. Low-dose hypersensitivity and increased radioresistance in a panel of human tumor cell lines with different radiosensitivity. Radiat. Res. 146, 399-413.

Xu, B., Kim, S.T., Lim, D.S., Kastan, M.B., 2002. Two molecularly distinct G(2)/M checkpoints are induced by ionizing irradiation. Mol. Cell Biol. 22, 1049-1059.

Zhou, H.N., Ivanov, V.N., Gillespie, J., Geard, C.R., Amundson, S.A., Brenner, D.J., Yu, Z.L., Lieberman, H.B., Hei, T.K., 2005. Mechanism of radiation-induced bystander effect: role of the cyclooxygenase-2 signaling pathway. Proc. Natl. Acad. Sci. Unit. States Am. 102, 14641. 